\title{
Incidência de dengue e indicadores socioeconômicos e entomológicos em Santos, São Paulo, 2012-2016
}

RESUMO | Objetivo: descrever incidência de dengue em Santos/SP e relacionar coeficiente de incidência (CI) de dengue com indicadores socioeconômicos e entomológicos de 2012-2016. Método: estudo epidemiológico, descritivo, ecológico dos casos confirmados de dengue, residentes em Santos, de 2012-2016, do Sistema de Informação de Agravos de NotificaçãoOnline; foram obtidos seis indicadores socioeconômicos da base de setores censitários do Instituto Brasileiro de Geografia e Estatística-2010; o sétimo do Índice Paulista de Vulnerabilidade Social-2010 e os indicadores entomológicos da Secretaria de Saúde; foi aplicada Correlação bivariada de Spearman (SPSS-Statistics $®$ ). Aprovado pelo Comitê de Ética em Pesquisa-CAAE n79776017.1.0000.5479. Resultados: de 2012-2016 ocorreram 16.451 casos, com Cl de 117,4 (2012) a 2.122,8 (2013) casos/100.000 habitantes, maior no sexo feminino e de 15-29 anos; os fatores socioeconômicos foram mais significativos entre 2015-2016; o Índice de Densidade de fêmeas Aedes aegypti apresentou maior correlação positiva. Conclusão: descreveu-se perfil epidemiológico/entomológico da dengue, apoiando gestores nas ações locais de controle.

Palavras-chaves: Dengue; Epidemiologia descritiva; Fatores socioeconômicos; Entomologia; Aedes.

\begin{abstract}
Objectives: describe incidence of dengue in Santos/SP and relate dengue incidence coefficient (IC) with socioeconomic and entomological indicators from 2012-2016. Methods: epidemiological, descriptive, ecological study of confirmed cases of dengue, resident in Santos, from 2012-2016, of the Online-Notifiable Diseases Information System; six socioeconomic indicators were obtained from census sectors base of Brazilian Institute of Geography and Statistics-2010; the seventh from the São Paulo Index of Social Vulnerability-2010 and the entomological indicators from the Health Department; Spearman's Bivariate Correlation (SPSS-Statistics $\AA$ ) was applied. Approved by the Research Ethics Committee-CAAE n79776017.1.0000.5479. Results: from 2012-2016 there were 16.451 cases, with IC from 117,4 (2012) to 2.122,8 (2013) cases/100.000 inhabitants, higher in females and between 15-29 years old; socioeconomic factors were more significant between 2015-2016; the Density Index of Aedes aegypti females showed a greater positive correlation. Conclusion: epidemiological/entomological profile of dengue was described, supporting managers in local control actions.
\end{abstract}

Keywords: Dengue; Epidemiology, Descriptive; Socioeconomic Factors; Entomology; Aedes.

RESUMEN | Objetivos: describir incidencia del dengue en Santos/SP y relacionar coeficiente de incidencia (CI) del dengue con indicadores socioeconómicos y entomológicos para 2012-2016. Métodos: estudio epidemiológico, descriptivo, ecológico de casos confirmados de dengue, residentes en Santos, desde 2012-2016, del Sistema de Información de Enfermedades de Notificación-Online; obtuvieron-se seis indicadores socioeconómicos desde base de sectores censales del Instituto Brasileño de Geografía y Estadística-2010, el séptimo del Índice de Vulnerabilidad Social de São Paulo-2010 y los indicadores entomológicos del Departamento de Salud. Aplicó-se Correlación Bivariada de Spearman (SPSS-Statistics $®$ ). Aprobado por el Comité de Ética en Investigación-CAAE n79776017.1.0000.5479. Resultados: desde 2012-2016 hubo 16.451 casos, con Cl de 117,4 (2012) a 2.122,8 (2013) casos/100.000 habitantes, mayor en mujeres y de 15-29 años; los factores socioeconómicos fueron más significativos entre 2015-2016; el Índice de Densidad de hembras Aedes aegypti mostró mayor correlación positiva. Conclusión: describió-se perfil epidemiológico/entomológico del dengue, apoyando a los gerentes en acciones de control local.

Palabras claves: Dengue; Epidemiología Descriptiva; Factores Socioeconómicos; Entomología; Aedes.

Silvia Domingues dos Santos

Enfermeira. Mestra em Saúde Coletiva pela Faculdade de Ciências Médicas da Santa Casa de São Paulo. Enfermeira do Grupo de Vigilância Epidemiológica de Santos/Secretaria de Estado da Saúde de São Paulo. Santos (SP). Brasil.

ORCID: 0000-0002-0249-3422

\section{Manoel Carlos S. de Almeida Ribeiro}

Médico. Doutor em Medicina Preventiva pela Universidade de São Paulo. Professor adjunto da Faculdade de Ciências Médicas da Santa Casa de São Paulo e Membro associado da International Epidemiological Association. São Paulo (SP). Brasil.

ORCID: 0000-0001-6827-4874
Recebido em: 01/12/2020

Aprovado em: 15/12/2020

INTRODUÇÃO

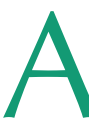
dengue é uma doença febril aguda, causada por vírus da família Flaviviridae, com quatro sorotipos DENV-1, DENV-2, DENV-3 e DENV-4. É sistêmica e apresenta amplo espectro clínico, de casos assintomáticos a graves, tendo a maioria evolução clínica benigna e parte evoluindo para formas graves, inclusive óbito ${ }^{1}$.

Mais da metade da população mundial vive sob risco de transmissão de dengue. A rápida e caótica urbanização, a globalização e as mudanças climáticas criaram um ambiente favorável ao desenvolvimento do mais importante vetor da dengue, o Aedes aegypti (Linnaeus) principalmente em regiões tropicais e subtropicais ${ }^{2}$.

A dengue atinge toda a sociedade, mas a carga pode ser maior nas comunidades pobres, com condições mais favoráveis ao vetor ${ }^{3}$. No Brasil as ações contra o Aedes aegypti incluem estratégias de controle como: saneamento do meio ambiente, ações educativas para eliminar o vetor e seus criadouros e o combate por controle biológico e químico. Porém, por mais de 30 anos o Brasil lida com a pro- 
liferação do vetor e as doenças por ele transmitidas, cujas ações de controle têm sido ineficientes ${ }^{4}$.

A Região Metropolitana da Baixada Santista apresenta clima favorável ao desenvolvimento do vetor, elevada densidade e movimentação populacional. Abriga o maior porto da América Latina, onde anualmente atracam mais de 4.000 navios, com grande fluxo de pessoas e produtos $^{5}$. No porto santista, em 1980, foram detectados os primeiros focos da reintrodução do Aedes aegypti no Estado, eliminados com medidas de controle eficazes ${ }^{6}$.

$\mathrm{Na}$ Baixada Santista o município de Santos foi o primeiro a apresentar transmissão de dengue, registrando 893 casos em 19977. De 1997 a junho de 2005, os casos de dengue residentes nesta região representaram 43,7\% dos casos do Estado ${ }^{5}$.

De 2012 a 2016 a Baixada Santista permaneceu entre as regiões de maior ocorrência de casos de dengue no Estado de São Paulo: Campinas (226.202), com 17,7\% dos casos do Estado; São José do Rio Preto (107.735); Ribeirão Preto (87.169), com 6,8\% dos casos; Piracicaba (86.791); Sorocaba (79.702); e Baixada Santista com 57.355 casos, 4,5\% dos casos do Estado 7 .

Em Santos, de 1997 a 2012, a curva da série histórica dos casos de dengue acompanhou a curva do Estado de São Paulo, sendo o município responsável por uma parcela expressiva do total de casos do Estado, variando de $13,9 \%$ a $48,1 \%$ entre os anos de 1997 a 1999, 2001 a 2002 e 2004 a 20056. De 2012 a 2016 Santos registrou 16.451 casos de dengue, $28,7 \%$ dos casos da região e 1,3\% dos casos do Estado. Neste período seu coeficiente de incidência foi superior ao estadual de 2012 a 20147, com média a alta incidência em todos os anos, conforme o Programa Nacional de Controle da Dengue (PNCD) ${ }^{8}$.

De 2006 a 2016 o coeficiente de incidência de casos prováveis de dengue apresentou tendência crescente no Brasil, variando de 212,1 (2009) a 825,8 (2015), no Estado de São Paulo, de 29,0 (2009) a 1.679,5 (2015), e em Santos, de 25,6
(2008) a 2.585,2 (2013) casos/100.000 habitantes ${ }^{9}$.

Considerando a importância da Região Metropolitana da Baixada Santista e de Santos no contexto das epidemias de dengue do Estado, é essencial o desenvolvimento de um estudo para caracterizar e analisar o perfil epidemiológico e entomológico da doença na cidade, contribuindo para a melhoria das estratégias locais de prevenção e controle da dengue. Assim, o objetivo deste estudo é descrever a incidência de dengue em Santos, segundo ano, sexo, faixa etária e relacionar coeficiente de incidência de dengue com indicadores socioeconômicos e entomológicos no período de 2012-2016.

\section{MÉTODOS}

Estudo epidemiológico descritivo e ecológico, cujos grupos de variáveis elencadas incluem incidência de casos confirmados de dengue, variáveis socioeconômicas e variáveis entomológicas. A população de estudo é composta pelos casos confirmados de dengue, residentes em Santos, notificados no Sistema de Informação de Agravos de Notificação-Online (SINAN-Online) no período de 2012 a 2016. Foram considerados casos confirmados os casos suspeitos confirmados: laboratorialmente por sorologia IgM, NS1 teste rápido ou Elisa (Ensaio de imunoabsorção enzimática), isolamento viral, RT-PCR (Transcrição reversa seguida de reação em cadeia da polimerase), imuno-histoquímica; e por critério clínico-epidemiológico nos períodos epidêmicos (exceto os primeiros casos da epidemia na área, que tem confirmação laboratorial) ${ }^{1}$.

Localizado no litoral do Estado de São Paulo, sede da Baixada Santista, Santos possui 281,0 km2, 419.400 habitantes e 1.494,26 habitantes/km2. Em 2017 apresentou taxa de mortalidade infantil de 9,0 óbitos/mil nascidos vivos, com Índice de Desenvolvimento Humano Municipal de 0,84010 (2010). Apresenta clima quente e úmido e economia pautada nas atividades portuária, comercial e turística ${ }^{6}$.
A base de dados referente aos casos de dengue foi extraída em 2017 do SINAN-Online pelo TabWin-Datasus V.3.6 (2010). O coeficiente de incidência (Cl) de dengue foi calculado por 100.000 habitantes, utilizando como denominador a população residente em Santos de 2012 a 2016, conforme dados disponíveis no Departamento de Informática do SUS (DATASUS $)^{11}$. Foi utilizada a população correspondente ao estratificar por sexo e faixa etária. A razão dos $\mathrm{Cl}$ de dengue por ano de início de sintomas, segundo faixa etária e sexo foi assim calculada: razão entre cada $\mathrm{Cl}$ por faixa etária, sexo e ano e o Cl da faixa etária de maiores de 60 anos, sexo e ano (esta última utilizada como denominador por ser a faixa etária com menor $\mathrm{Cl}$ no período, adotada como referência para comparação relativa). Para classificar a Incidência de dengue foram adotados os parâmetros do PNCD ( ${ }^{\circ}$ casos $/ 100.000$ habitantes): Baixa $\leq 100$; Média $>100$ e $<$ 300; Alta $\geq 3008$.

Seis indicadores socioeconômicos foram obtidos da base de setores censitários de Santos, conforme o Censo de 2010 do Instituto Brasileiro de Geografia e Estatística (IBGE) ${ }^{12}$ e agregados pelos 65 bairros, sendo assim calculados:

- Densidade demográfica (habitantes/ $\mathrm{km}^{2}$ ): divisão da população do bairro pela área do bairro em Km2 (calculada no TerraView V.4.2.2).

- Proporção de pessoas residentes alfabetizadas com idade maior ou igual ( $\geq$ ) 5 anos: porcentagem de pessoas residentes no bairro alfabetizadas com idade maior ou igual ( $\geq$ ) 5 anos em relação à população total do bairro.

- Proporção de domicílios com renda per capita até meio (1/2) salário mínimo; Proporção de domicílios com abastecimento de água da rede geral; Proporção de domicílios com esgotamento sanitário via rede geral de esgoto; Proporção de domicílios com lixo coletado: porcentagem de cada indicador em 
relação ao total de domicílios particulares permanentes do bairro.

O sétimo indicador foi obtido do Índice Paulista de Vulnerabilidade Social (IPVS) 2010/Fundação Sistema Estadual de Análise de Dados (Seade), baseado em dados de escolaridade, saúde, arranjo familiar, inserção no mercado de trabalho, bens e serviços públicos ${ }^{13}$.

Agregou-se a base de dados do IPVS por bairro, adotando-se a Proporção de pessoas residentes em setores censitários com IPVS 4, 5 ou 6 (Vulnerabilidade média, alta ou muito alta) em relação à população total do bairro.

Os indicadores entomológicos referentes ao período de 2012 a 2016 foram extraídos em 2017 dos bancos de dados dos índices de infestação vetorial da Seção de Controle de Vetores (SECOVE) - Secretaria Municipal de Saúde de Santos.

O Índice de Densidade Larvária (IDL) é composto pelo Índice de Breteau (IB), indicador adotado para a avaliação de densidade larvária no Estado de São Paulo6, sendo assim calculado ${ }^{14}$ :

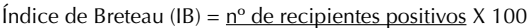
$\mathrm{n}^{\circ}$ de imóveis pesquisados

Em Santos, a SECOVE avalia o IB em janeiro, abril, julho e outubro, com duas exceções: em abril de 2013 não foi avaliado devido à epidemia de dengue; e em 2014 foi avaliado em fevereiro, abril, julho e outubro. Em 2012, o município adotou o "Monitoramento Inteligente da Dengue" (Ml-Dengue) como ferramenta para monitoramento contínuo da infestação do Aedes aegypti e direcionamento das ações de controle. O MI-Dengue é composto por armadilhas (MosquiTRAP ${ }^{\circledR}$ ) que capturam fêmeas adultas de Aedes aegypti (principalmente fêmeas grávidas), associadas a um sistema informatizado de coleta, transmissão e acesso das informações ${ }^{15}$.

Em janeiro de 2012 foram instaladas 461 armadilhas no município, distantes 250m entre elas, com distribuição média de 16 armadilhas $/ \mathrm{km} 2$. Cada agente de endemias vistoria 78 armadilhas/semana, contabilizando as fêmeas de Aedes aegypti capturadas.

Os indicadores entomológicos foram agregados por ano e bairro, em janeiro, abril, julho e outubro, sendo assim calculados:

- Índice de Densidade Larvária (IDL): razão entre o número de recipientes positivos para Aedes aegypti e o número de imóveis pesquisados multiplicado por 100.

- Índice de Densidade de fêmeas de Aedes aegypti (IDF): razão entre o número de fêmeas adultas de Aedes aegypti capturadas e o número de armadilhas.

Para a análise estatística dos dados foi utilizada Correlação bivariada de Spearman com SPSS Statistics ${ }^{\circledR}$ 22.0.0.0 (2014) e nível de significância de 5\% para relacionar o $\mathrm{Cl}$ de dengue com indicadores socioeconômicos e indicadores entomológicos no período de 2012 a 2016. Agregou-se o $\mathrm{Cl}$ de dengue por mês de início de sintomas e por bairro (por 10.000 habitantes), utilizando como denominador a população residente/bairro, conforme o Censo 2010 do IBGE ${ }^{12}$.

Os bairros da Área Portuária (sem po-

Figura 1 - Coeficiente de incidência de dengue (por 100.000 habitantes) por ano epidemiológico de início de sintomas, Santos, São Paulo. 2012-2016.

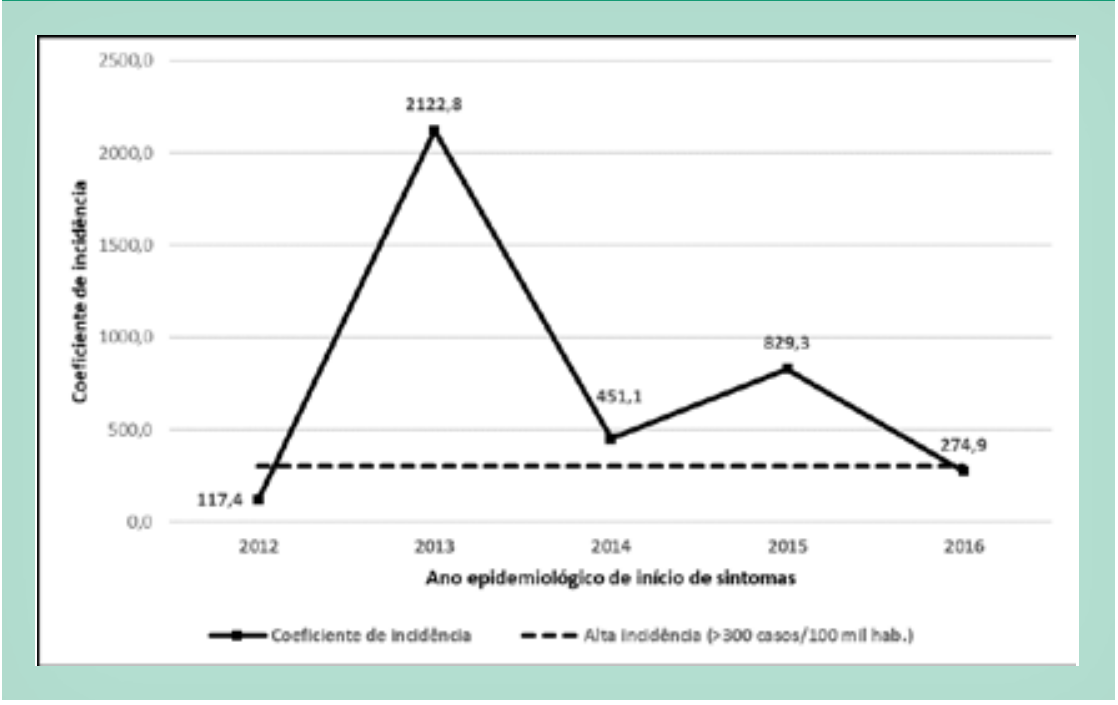

Fontes: SINAN-Online, 2012-2016; DATASUS, 2012-2016. pulação residente) e Morros Cachoeira, Chico de Paula e Embaré foram excluídos por apresentarem população menor que 200 habitantes; assim como os bairros da densidade demográfica (13,5 habitantes/ $\mathrm{km}^{2}$ ) do município ${ }^{12}$. Portanto, há poucos casos de dengue nestes bairros/áreas, mas devido à pequena/ausente população residente apenas 1 caso geraria alta incidência, ocasionando "outliers" na análise estatística.

Os pesquisadores comprometem-se a manter dados confidenciais, conforme Resoluções 196/1996 e 466/2012 $2^{16-17}$. O Projeto de pesquisa (CAAE n79776017.1.0000.5479) foi aprovado em 20/02/18 no Comitê de Ética em Pesquisa da Santa Casa de Misericórdia de São Paulo.

\section{RESULTADOS}

De 2012 a 2016 foram registrados 16.451 casos confirmados de dengue em Santos, com dois picos epidêmicos: em 2013 com 9.195 casos $(2.122,8$ casos/100.000 habitantes); e em 2015 com 3.599 casos (829,3 casos/100.000 habitantes) (Figura 1). Área continental, que apresenta a menor 
Em relação à circulação viral, houve uma mudança gradativa do sorotipo DENV-4 para DENV-1 ao longo do período. Em 2012, 100\% dos casos sorotipados eram DENV-4. Nos anos subsequentes a proporção do sorotipo DENV-1 começou a crescer até que em 2015 passou a ser 100,0\% dos casos sorotipados. Em 2016 não foram registrados sorotipos no SINAN-Online.

Tabela 1 - Coeficientes de incidência de dengue (por 100.000 habitantes) e razão dos coeficientes de incidência de dengue por ano epidemiológico de início de sintomas, segundo faixa etária e sexo, Santos, São Paulo. 2012-2016.

\begin{tabular}{|c|c|c|c|c|c|c|c|c|c|c|c|}
\hline \multirow{3}{*}{\multicolumn{2}{|c|}{$\begin{array}{l}\text { Sexo/Faixa Etária } \\
\text { (anos) }\end{array}$}} & \multicolumn{10}{|c|}{ Ano epidemiológico de início de sintomas/Indicador } \\
\hline & & \multicolumn{2}{|c|}{2012} & \multicolumn{2}{|c|}{2013} & \multicolumn{2}{|c|}{2014} & \multicolumn{2}{|c|}{2015} & \multicolumn{2}{|c|}{2016} \\
\hline & & $\mathrm{Cl}$ & $\mathrm{RCl}$ & $\mathrm{Cl}$ & $\mathrm{RCl}$ & $\mathrm{Cl}$ & $\mathrm{RCl}$ & $\mathrm{Cl}$ & $\mathrm{RCl}$ & $\mathrm{Cl}$ & $\mathrm{RCl}$ \\
\hline \multirow{4}{*}{ Feminino } & $0-14$ & 94,4 & 2,0 & 1957,5 & 1,7 & 320,5 & 1,1 & 711,3 & 1,6 & 299,6 & 2,1 \\
\hline & $15-29$ & 153,9 & 3,2 & 3376,8 & 2,9 & 633,0 & 2,1 & 1570,0 & 3,6 & 415,4 & 3,0 \\
\hline & $30-59$ & 168,7 & 3,5 & 2548,8 & 2,2 & 660,9 & 2,2 & 1006,8 & 2,3 & 328,8 & 2,3 \\
\hline & $\geq 60$ & 48,2 & 1,0 & 1173,7 & 1,0 & 300,5 & 1,0 & 432,7 & 1,0 & 140,1 & 1,0 \\
\hline \multirow{4}{*}{ Masculino } & $0-14$ & 128,0 & 2,6 & 1788,7 & 1,7 & 353,8 & 2,1 & 635,4 & 1,9 & 324,3 & 4,1 \\
\hline & $15-29$ & 133,3 & 2,7 & 2965,7 & 2,9 & 523,7 & 3,1 & 1233,0 & 3,7 & 349,2 & 4,4 \\
\hline & $30-59$ & 103,6 & 2,1 & 1804,5 & 1,8 & 394,8 & 2,4 & 673,8 & 2,0 & 246,0 & 3,1 \\
\hline & $\geq 60$ & 49,5 & 1,0 & 1025,3 & 1,0 & 166,6 & 1,0 & 330,5 & 1,0 & 79,9 & 1,0 \\
\hline
\end{tabular}

Tabela 2 - Coeficiente de correlação de Spearman entre coeficiente de incidência de dengue (por 10.000 habitantes) por ano e indicadores socioeconômicos, por bairros, Santos, São Paulo. 2012-2016.

\begin{tabular}{|c|c|c|c|c|c|}
\hline \multirow{2}{*}{ Indicadores socioeconômicos } & \multicolumn{5}{|c|}{ Coeficiente de incidência de dengue } \\
\hline & 2012 & 2013 & 2014 & 2015 & 2016 \\
\hline Densidade demográfica (habitantes $/ \mathrm{km}^{2}$ ) & $-0,104$ & $-0,176$ & $-0,051$ & $-0,261$ & $-0,389\left(^{*}\right)$ \\
\hline Proporção de alfabetização (idade $\geq 5$ anos) & $-0,262$ & $-0,296\left(^{*}\right)$ & $-0,057$ & $-0,435\left(^{*}\right)$ & $-0,356\left(^{*}\right)$ \\
\hline $\begin{array}{l}\text { Proporção de renda per capita até } 1 / 2 \\
\text { salário mínimo }\end{array}$ & 0,246 & 0,201 & 0,005 & $0,384\left(^{*}\right)$ & $0,324\left(^{*}\right)$ \\
\hline $\begin{array}{l}\text { Proporção de abastecimento de água da } \\
\text { rede geral }\end{array}$ & 0,004 & $-0,111$ & 0,120 & $-0,208$ & $-0,141$ \\
\hline $\begin{array}{l}\text { Proporção de esgotamento sanitário da } \\
\text { rede geral }\end{array}$ & $-0,235$ & $-0,142$ & $-0,053$ & $-0,352\left(^{*}\right)$ & $-0,393\left(^{*}\right)$ \\
\hline Proporção de lixo coletado & $-0,137$ & 0,056 & $-0,046$ & $-0,104$ & 0,017 \\
\hline $\begin{array}{l}\text { Proporção de pessoas residentes em } \\
\text { setores censitários com IPVS } 4,5 \text { ou } 6\end{array}$ & 0,151 & 0,210 & 0,028 & $0,332\left(^{*}\right)$ & 0,237 \\
\hline
\end{tabular}

*Correlações significativas $(p<0,05)$

IPVS = Índice Paulista de Vulnerabilidade Social; CC = Coeficiente de correlação

Fontes: SINAN-Online, 2012-2016; Fundação Seade - Índice paulista de vulnerabilidade social, 2010; Censo IBGE, 2010. de 60 anos) constituíram o grupo de menor incidência para ambos os sexos durante todo o período. Ao compararmos os grupos etários de maior incidência (1529 anos e 30 a 59 anos) com os idosos, evidenciamos um risco para os primeiros aproximadamente 2 a 3 vezes maior. Chama a atenção ainda o risco 4 vezes maior nas faixas etárias mais jovens $(0$ a 14 anos e 15-29 anos) no ano de 2016 para sexo masculino (Tabela 1 ).

De acordo com a Tabela 2, a investigação da correlação entre os indicadores socioeconômicos e coeficiente de incidência por bairro no período demonstrou que para os anos de 2012 a 2014 não existe um padrão de correlação entre as variáveis. Por outro lado, em 2015 e 2016 foram identificadas correlações estatisticamente significativas de intensidade fraca e moderada, das quais destacamos:

- Correlação negativa com densidade demográfica em 2016 (-0,389).

- Correlação negativa com Proporção de alfabetização da população com idade maior ou igual $(\geq)$ 5 anos em $2015(-0,435)$ e 2016 $(-0,356)$.

- $\quad$ Correlação positiva com Proporção de domicílios com renda per capita até meio $(1 / 2)$ salário mínimo em $2015(0,384)$ e $2016(0,324)$.

- Correlação negativa com Proporção de domicílios com esgoto em $2015(-0,352)$ e $2016(-0,393)$.

- Correlação positiva com proporção de pessoas residentes em setores censitários com maior vulnerabilidade - IPVS 4, 5 ou 6 em 2015 $(0,332)$.

Os indicadores de coleta de lixo e abastecimento de água não se correlacionaram com os coeficientes de incidência (Tabela 2).

O estudo mostrou sazonalidade da dengue em abril. Nos anos epidêmicos os picos de abril foram 700,3 (2013) e 434,9 (2015) casos/100.000 habitantes. O IDF acompanhou os picos do $\mathrm{Cl}$ em abril 
de $2012(3,3), 2015(7,5)$ e $2016(7,0)$ e seus menores valores ocorreram em julho (exceto em 2012 quando o menor valor do IDF foi verificado em janeiro). O IDL acompanhou os picos do $\mathrm{Cl}$ em abril de $2012(0,8), 2014(3,0)$ e 2015 (1,9); e em julho foram registrados seus menores valores (com valores mínimos também em outubro de 2012 e 2013) (Tabela 3).

Conforme e Tabela 4, a análise da correlação entre os indicadores entomológicos (IDL e IDF) e o $\mathrm{Cl}$ de dengue por bairro evidenciou poucas correlações estatisticamente significativas de intensidade moderada. O IDF apresentou maior correlação positiva em relação ao $\mathrm{Cl}$ quando comparado ao IDL, porém não significativa na maioria dos anos. Em suma, o Cl correlacionou-se com significância estatística com:

- IDL no terceiro trimestre de 2012 $(-0,330)$.

- IDF e IDL no primeiro trimestre de $2013(0,412$ e 0,319, respectivamente).

- $\quad$ IDF no último trimestre de 2015 $(0,390)$.

\section{DISCUSSÃO}

O padrão temporal da incidência da Dengue em Santos no período estudado foi semelhante ao padrão estadual e ao padrão da Baixada Santista ${ }^{7}$. Não foi identificado um padrão cíclico (com surtos em determinados anos18), sendo que nos anos de 2013 a 2015 a incidência se manteve alta, conforme o $\mathrm{PNCD}^{8}$, acima dos 300 casos/100.000 habitantes e com picos expressivos particularmente nos anos de 2013 e 2015. Este padrão deve estar associado à mudança da predominância do sorotipo DENV-4 para DENV-1 ao longo do período estudado.

\begin{tabular}{|c|c|c|c|c|c|c|c|c|c|c|c|c|c|c|c|c|c|c|c|c|}
\hline \multirow{3}{*}{ Indicador } & \multicolumn{20}{|c|}{ Mês/Ano de início de sintomas/captura/avaliação } \\
\hline & \multicolumn{4}{|c|}{2012} & \multicolumn{4}{|c|}{2013} & \multicolumn{4}{|c|}{2014} & \multicolumn{4}{|c|}{2015} & \multicolumn{4}{|c|}{2016} \\
\hline & jan & $\mathrm{abr}$ & jul & out & jan & abr & jul & out & fev & abr & jul & out & jan & abr & jul & out & jan & abr & jul & out \\
\hline $\mathrm{Cl}$ & 0,5 & 32,0 & 10,3 & 1,2 & 50,5 & 700,3 & 1,4 & 0,2 & 4,8 & 69,1 & 10,3 & 4,3 & 2,9 & 434,9 & 8,6 & 1,0 & 5,2 & 105,9 & 0,7 & 1,0 \\
\hline IDF & 0,8 & 3,3 & 1,7 & 1,8 & 3,1 & 2,9 & 2,0 & 2,0 & 3,4 & 2,9 & 2,5 & 3,5 & 5,4 & 7,5 & 3,0 & 3,3 & 5,1 & 7,0 & 1,7 & 1,8 \\
\hline IDL & 0,7 & 0,8 & 0,4 & 0,4 & 1,5 & $-{ }^{*}$ & 0,2 & 0,2 & 1,9 & 3,0 & 0,8 & 1,8 & 1,8 & 1,9 & 0,7 & 1,5 & 1,5 & 1,3 & 0,3 & 0,6 \\
\hline
\end{tabular}

*Em abril de 2013 o IDL não foi avaliado devido à epidemia de dengue.

$\mathrm{Cl}$ = Coeficiente de incidência; IDF = Índice de Densidade de fêmeas de Aedes Aegypti; IDL = Índice de Densidade Larvária

Fontes: SINAN-Online, 2012-2016; Censo IBGE, 2010; Índices de infestação vetorial - SECOVE/Secretaria Municipal de Saúde de Santos, $2012-2016$.

Tabela 4 - Coeficiente de correlação de Spearman entre coeficiente de incidência de dengue (por 10.000 habitantes) e Índice de Densidade de fêmeas de Aedes aegypti e Índice de Densidade Larvária - meses de janeiro "jan", abril "abr", julho "jul" e outubro "out", por bairro, Santos, São Paulo. 2012-2016.

\begin{tabular}{|c|c|c|c|c|c|c|}
\hline \multirow{2}{*}{\multicolumn{2}{|c|}{$\begin{array}{l}\text { Indicador/ } \\
\text { Mês }\end{array}$}} & \multicolumn{5}{|c|}{$\begin{array}{l}\text { Coeficiente de incidência de dengue } \\
\text { (jan, abr, jul, out) }\end{array}$} \\
\hline & & 2012 & 2013 & 2014 & 2015 & 2016 \\
\hline \multirow{4}{*}{ IDF } & jan & 0,286 & $0,412\left(^{*}\right)$ & 0,132 & $-0,255$ & $-0,190$ \\
\hline & $a b r$ & 0,168 & $-0,084$ & 0,128 & 0,075 & 0,179 \\
\hline & jul & $-0,245$ & 0,161 & 0,035 & $-0,106$ & 0,044 \\
\hline & out & 0,152 & $-0,082$ & 0,177 & $0,390\left(^{*}\right)$ & 0,046 \\
\hline \multirow{4}{*}{ IDL } & jan & 0,149 & $0,319\left(^{*}\right)$ & 0,073 & $-0,249$ & $-0,106$ \\
\hline & $a b r$ & 0,018 & $-* *$ & 0,202 & 0,199 & 0,171 \\
\hline & jul & $-0,330\left(^{*}\right)$ & $-0,199$ & 0,127 & $-0,201$ & $-0,191$ \\
\hline & out & $-0,142$ & $-0,220$ & $-0,192$ & $-0,015$ & 0,198 \\
\hline
\end{tabular}

*Correlações significativas $(p<0,05)$

**Em abril de 2013 o IDL não foi avaliado devido à epidemia de dengue.

IDF = Índice de Densidade de fêmeas de Ae. Aegypti; IDL = Índice de Densidade Larvária

Fontes: SINAN-Online, 2012-2016; Censo IBGE, 2010; Índices de infestação vetorial - SECOVE/Secretaria Municipal de Saúde de Santos, 2012-2016.
Segundo a literatura, em Santos identificou-se o sorotipo DENV-1 em 1997 e os demais sorotipos em 1998 (DENV-2), em 2002 (DENV-3) e em 2012 (DENV-4). Em 1998 houve epidemia em Santos após introdução do DENV-1 em 1997; em 1999, após introdução do DENV-2 em 1998; e em 2002, quando houve registro do DENV-36. Por sua vez, a identificação de um novo sorotipo, DENV-4, em 2012 (100,0\%) com predomínio em 2013 (96,6\%) culminou na epidemia de dengue de 2013 que apresentou o maior $\mathrm{Cl}(2.122,8$ casos/100.000 habitantes) do período estudado.

De forma consistente, ao longo deste período, a incidência de Dengue em Santos foi maior no sexo feminino e na faixa etária de 15-29 anos. Este é um padrão referido em outros estudos, destacando-se os estudos realizados em São José do Rio Preto, de 1990 a $2005^{19}$, e em Araraquara, 
de 1991 a $2015^{20}$, nos quais também houve predomínio do agravo nas mulheres.

O maior acometimento das mulheres pode estar relacionado à influência do ambiente domiciliar/peridomiciliar na transmissão das doenças transmitidas pelo Aedes aegypti ${ }^{21}$, assim como a maior procura por assistência médica pelas muIheres, resultando em maior número de diagnósticos e notificações, o que pode acarretar um viés nas comparações entre as taxas encontradas ${ }^{22}$.

As altas incidências de dengue nos anos epidêmicos trazem uma carga de doença importante para a sociedade. Ao afetar principalmente as faixas etárias componentes da população economicamente ativa (o $\mathrm{Cl}$ foi maior nas faixas etárias de 15-29 anos, seguida pela de 30-59 anos), a dengue impacta na produtividade e gera ônus econômico em anos epidêmicos ${ }^{2}$.

A análise da correlação dos indicadores socioeconômicos estudados com a incidência de dengue a nível dos bairros em Santos revelou que existem correlações estatisticamente significantes mas elas são fracas ou moderadas e que este padrão não é verificado de forma consistente em todo o período. De modo geral, piores condições socioeconômicas se correlacionaram com maiores coeficientes de incidência de dengue. Uma hipótese seria que nas áreas com piores níveis socioeconômicos as características de infra-estrutura e saneamento básico oferecem melhores condições à proliferação do vetor. Em São José do Rio Preto - em estudo baseado em renda, instrução, analfabetismo e número de moradores por domicílio - houve associação entre risco de dengue e níveis socioeconômicos em 1994 e $1995^{23}$.

Cabe ainda o destaque para a densidade demográfica com algumas correlações negativas com o Cl. Na literatura existem poucos estudos sobre avaliação intra-urbana da dengue para tal discussão, no entanto, uma hipótese seria que, em Santos, a orla da praia e proximidades apresentam as maiores densidades demo- gráficas assim como os melhores indicadores socioeconômicos ${ }^{12-13}$.

O estudo mostrou sazonalidade da dengue em abril. Em Araraquara, de 1991 a 2015, o Cl teve pico entre março e maio ${ }^{20}$. Casos de dengue também foram registrados em Santos nos demais meses, mostrando endemização da doença, com ocorrência durante todo o $\mathrm{ano}^{24}$. Ainda assim, a identificação da sazonalidade contribui no planejamento das atividades de vigilância epidemiológica e controle de vetores, intensificando esforços antes deste período para minimização da dispersão viral ${ }^{20}$.

Os indicadores entomológicos IDF e IDL demonstraram um comportamento aparentemente associado à evolução temporal do coeficiente de incidência de dengue acompanhando os picos do $\mathrm{Cl}$ em abril em diversos anos do período estudado. No entanto, as correlações entre os indicadores entomológicos e o coeficiente de incidência de dengue por bairro não expressaram um padrão bem definido. Nos vinte períodos investigados apenas em 2 para IDL e 2 para IDF as correlações foram estatisticamente significantes. Em estudo em São José do Rio Preto, de 2012 a 2013, todavia, o aumento dos casos de dengue ocorreu paralelamente ao aumento do número de fêmeas adultas de Aedes aegypti ${ }^{25}$.

Em relação ao IDL é preciso considerar ainda o lag existente entre o aumento da população larvária e o aumento de casos, visto que somente fêmeas adultas transmitem o vírus ${ }^{25}$. Em Araraquara, de 1991 a 2015, a curva dos casos aumentou um ou dois meses após pico do índice larvário ${ }^{20}$.

A fragilidade das correlações dos indicadores entomológicos pode estar relacionada à escolha do bairro como unidade espacial, diante de sua heterogeneidade, sendo assim uma das limitações do estudo. Ao apresentar dados socioeconômicos e de saúde agregados em áreas abrangentes, as análises podem limitar-se à situação média dos indivíduos, impossibilitando identificar a heterogeneidade existente ${ }^{26}$.
O uso dos dados provenientes do SINAN-Online consiste em outra limitação do estudo, pelo viés gerado pela subnotificação, cobertura e qualidade dos dados.

É necessário considerar como outra limitação o estudo do tipo ecológico, delineamento que levanta hipóteses para estudos analíticos com maior poder para comprovar relações causais. Assim, sugere-se estudos futuros com correlações multivariadas e cluster tempo-espacial, e possível redução da unidade espacial visando identificar áreas mais homogêneas, buscando maior compreensão dos níveis de risco à dengue no espaço intra-urbano.

\section{CONCLUSÃO}

O estudo descreveu o perfil epidemiológico da dengue em Santos, com padrões semelhantes aos perfis do estado de São Paulo e da Baixada Santista. Também mostrou diferenciais intra-urbanos - que contribuem para o entendimento da dinâmica local das epidemias - e relacionou a doença a indicadores socioeconômicos e entomológicos, com análises locais inéditas do Índice de Densidade de fêmeas de Aedes aegypti (IDF).

Em relação aos indicadores socioeconômicos considerados para o nível de agregado do estudo (bairro) foram apontadas relações entre áreas com piores condições socioeconômicas e maior coeficiente de incidência de dengue, principalmente no que se refere aos anos de 2015 e 2016. A literatura diverge sobre a associação entre condições socioeconômicas e a ocorrência de dengue, destacando assim a complexidade desta relação. No entanto, o estudo relacionou a dengue com fatores socioeconômicos indo de encontro à temática das desigualdades sociais em saúde e o perfil epidemiológico dos diferentes grupos sociais, apontando também a relevância de buscar formas de enfrentamento no âmbito das políticas públicas sociais.

Por sua vez, embora o estudo tenha mostrado relações entre os indicadores entomológicos e a incidência de dengue, 
estas não são capazes de explicar ou prever de forma eficiente a variação da incidência da doença.

Ainda assim, os resultados contribuem para planejar atividades de vigilân- cia epidemiológica e controle de vetores, com intervenções focais no período que antecede a sazonalidade - visando reduzir a infestação - e uma vigilância epidemiológica eficaz para reconhecer precocemente a transmissão. Além disso, a importância do monitoramento do vetor adulto pode validar a manutenção do "Monitoramento Inteligente da Dengue" em Santos.

\section{Referências}

1. Ministério da Saúde (BR). Secretaria de Vigilância em Saúde. Coordenação-Geral de Desenvolvimento da Epidemiologia em Serviços. Guia de Vigilância em Saúde. 3. ed. Brasília: Ministério da Saúde; 2019. Capítulo 7, Dengue, Chikungunya e Zika; p. 413-421.

2. Castro MC, Wilson ME, Bloom DE. Disease and economic burdens of dengue. Lancet Infect Dis [Internet]. 2017 Mar [cited 2020 mai 23];17(3):e70-78. Available from: https://www.sciencedirect.com/science/article/abs/pii/ S147330991630545X. doi: 10.1016/S1473-3099(16)30545-X

3. World Health Organization. Dengue guidelines for diagnosis, treatment, prevention and control [Internet]. World Health Organization; 2009 [cited 2020 mai 23] Available from: http://www.who.int/tdr/publications/documents/dengue-diagnosis.pdf

4. Ministério da Saúde (BR). Secretaria de Vigilância em Saúde. Departamento de Vigilância Epidemiológica. Diretrizes nacionais para prevenção e controle de epidemias de dengue. 1. ed. Brasília: Ministério da Saúde; 2009.

5. Superintendência de Controle de Endemias (SUCEN). Coordenadoria de Controle de Doenças (CCD). Dengue: ações de combate aos vetores. Rev Saude Publica [Internet]. 2005 [citado 2020 mai 23];39(6):985-6. Disponível em: https:// www.scielosp.org/pdf/rsp/2005.v39n6/985-986

6. Fernandes MAB, Natal D, Domingos MF. Aspectos epidemiológicos da transmissão de dengue em Santos, São Paulo, no período de 1997 a 2012. J Health Biol Sci [Internet]. 2014 mar [citado 2020 set 29];2(1):5-12. Disponível em: https://www.researchgate.net/publication/275505500_ASPECTOS_EPIDEMIOLOGICOS_DA_TRANSMISSAO_DE_DENGUE_EM_SANTOS_SAO_PAULO_NO_ PERIODO_DE_1997_A_2012 doi: 10.12662/2317-3076jhbs.v2i1.45.p.5.2014

7. Centro de Vigilância Epidemiológica. Casos autóctones de dengue por DIR e município [Internet]. 2016. São Paulo: Centro de Vigilância Epidemiológica; 2016 [citado 2020 jul 18]. Disponível em: http://www.saude.sp.gov.br/cve-centro-de-vigilancia-epidemiologica-prof-alexandre-vranjac/areas-de-vigilancia/ doencas-de-transmissao-por-vetores-e-zoonoses/arboviroses-urbanas/dengue/ dados-estatisticos

8. Ministério da Saúde (BR). Fundação Nacional de Saúde. Programa Nacional de Controle da Dengue. Brasília: Ministério da Saúde; 2002.

9. Brasil. Ministério da Saúde. Departamento de Informática do SUS. Informações de Saúde - TABNET - Epidemiológicas e Morbidade [Internet]. 2016. Braślia: Departamento de Informática do SUS; 2016 [citado 2020 abr 28]. Disponível em: http://www2.datasus.gov.br/DATASUS/index.php?area=0203\&id=29878153 10. Instituto Brasileiro de Geografia e Estatística. Panorama. Município de Santos. [Internet]. 2020. Rio de Janeiro: Instituto Brasileiro de Geografia e Estatística; 2020 [citado 2020 abr 28]. Disponível em: https://cidades.ibge.gov.br/brasil/ sp/santos/panorama

11. Brasil. Ministério da Saúde. Departamento de Informática do SUS-Datasus. Informações de Saúde - TABNET - Demográficas e Socioeconômicas [Internet]. 2016. Brasília: Departamento de Informática do SUS; 2016 [citado 2020 abr 28]. Disponível em: http://www2.datasus.gov.br/DATASUS/index.php?area=0206

12. Instituto Brasileiro de Geografia e Estatística. Censo Demográfico - Censo 2010. [Internet]. 2010. Rio de Janeiro: Instituto Brasileiro de Geografia e Estatística; 2010 [citado 2020 abr 28]. Disponível em: http://www.censo2010.ibge. gov.br/sinopseporsetores/.

13. Fundação Seade. Índice paulista de vulnerabilidade social. Principais resultados. [Internet]. 2010. São Paulo: Fundação Seade; 2010 [citado 2020 abr 28]. Disponível em: http://www.ipvs.seade.gov.br/view/pdf/ipvs/principais_resultados.pdf

14. Ministério da Saúde (BR). Secretaria de Vigilância em Saúde. Departamento de Vigilância das Doenças Transmissíveis. Levantamento Rápido de Índices para
Ae. aegypti (LIRAa) para vigilância entomológica do Aedes aegypti no Brasil: metodologia para avaliação dos índices de Breteau e Predial e tipo de recipientes. 2. ed. Brasília: Ministério da Saúde; 2013.

15. Eiras ÁE, Resende MC. Preliminary evaluation of the "Dengue-MI" technology for Aedes aegypti monitoring and control. Cad Saude Publica [Internet]. 2009 [cited 2020 Mai 23];25(S):45-58. Available from: https://pdfs.semanticscholar. org/6e7e/2babaac1bd88855a12ef56fb425749bf547f.pdff

16. Brasil. Resolução ${ }^{\circ} 196$, de 10 de outubro de 1996. Aprova as diretrizes e normas regulamentadoras de pesquisas envolvendo seres humanos [Internet]. [citado 2020 abr 28]. Disponível em: https://www.nescon.medicina.ufmg.br/biblioteca/imagem/1944.pdf

17. Brasil. Resolução $n^{\circ} 466$, de 12 de dezembro de 2012. Aprova as diretrizes e normas regulamentadoras de pesquisas envolvendo seres humanos [Internet]. [citado 2020 abr 28]. Disponível em: https://portal.fiocruz.br/sites/portal.fiocruz. br/files/documentos/res_cns_466.2012_-_revoga_196.pdf

18. Descloux $E$, Mangeas $M$, Menkes $C E$, Lengaigne $M$, Leroy $A$, Tehei $T$, et al. Climate-Based Models for Understanding and Forecasting Dengue Epidemics. PLoS Negl Trop Dis [Internet]. 2012 Feb [cited 2020 mai 23];6(2):e1470. Available from: https://www.ncbi.nlm.nih.gov/pubmed/22348154. doi: 10.1371/journal. pntd.0001470

19. Scandar SAS, Vieira P, Junior RPC, Silva RA, Papa M, Sallum MAM. Dengue em São José do Rio Preto, ESP, 1990 a 2005. Bepa [Internet]. 2010 [citado 2020 mai 23];7(81):4-16. Disponível em: http://periodicos.ses.sp.bvs.br/pdf/bepa/v7n81/ v7n81a01.pdf

20. Ferreira AC, Chiaravalloti-Neto F, Mondini A. Dengue em Araraquara, SP: epidemiologia, clima e infestação por Aedes aegypti. Rev Saude Publica [Internet]. 2018 fev [citado 2020 abr 28];52:18. Disponível em: http://www.revistas.usp.br/ rsp/article/view/143840. doi: 10.11606/S1518-8787.2018052000414

21. Moura RF, Almeida AB. Análise espacial dos casos confirmados de Zika Vírus no estado de São Paulo, Brasil. Nursing (São Paulo) [Internet]. 2020 jun [citado 2020 nov 20];23(265): 4107-11. Disponível em: https://pesquisa.bvsalud.org/portal/resource/pt/biblio-1117642 doi: 10.36489/nursing.2020v23i265p4107-4111 22. Cavalcante WD, Vilar MSA, Vilar DA, Soares CS. Características epidemiológicas da dengue na comunidade São Januário II na cidade de Campina Grande - PB. Rev Bras Farm [Internet]. 2011 [citado 2020 Mai 27];92(4):287-94. Disponivel em: http://www.rbfarma.org.br/files/rbf-2011-92-4-7-287-294.pdf 23. Mondini A, Chiaravalloti-Neto F. Socioeconomic variables and dengue transmission. Rev Saude Publica [Internet]. 2007 [cited 2020 mai 23];41(6):923-30. Available from: http://www.scielo.br/pdf/rsp/v41n6/6079.pdf

24. Mondini A, Chiaravalloti-Neto F, Gallo YSM, Lopes JCC. Análise espacial da transmissão de dengue em cidade de porte médio do interior paulista. Rev Saude Publica [Internet]. 2005 jun [citado 2020 Jul 20];39(3):444-51. Disponivel em: http://www.scielo.br/scielo.php?script=sci_arttext\&pid=S0034-89102005000300016\&lng=en. https://doi.org/10.1590/ S0034-89102005000300016.

25. Parra MCP, Fávaro EA, Dibo MR, Mondini A, Eiras AE, Kroon EG, et al. Using adult Aedes aegypti females to predict areas at risk for dengue transmission: a spatial case-control study. Acta Tropica [Internet]. 2018 Jun [cited 2020 mai 23];185(0):43-53. Available from: http://www.sciencedirect.com/science/article/ pii/S0001706X17312020. doi: 10.1016/j.actatropica.2018.02.018

26. Akerman M, Campanario P, Maia PB. Saúde e meio ambiente: análise de diferenciais intra-urbanos, Município de São Paulo, Brasil. Rev Saude Publica [Internet]. 1996 [citado 2020 mai 23];30(4):372-82. Disponivel em: https://www. scielosp.org/article/ssm/content/raw/?resource_ssm_path=/media/assets/rsp/ v30n4/5088.pdf 